\title{
New Pancreatic Cancer Biomarkers eIF1, eIF2D, eIF3C and eIF6 Play a Major Role in Translational Control in Ductal Adenocarcinoma
}

\author{
NICOLE GOLOB-SCHWARZL ${ }^{1,2}$, PHILIP PUCHAS ${ }^{1}$, MARGIT GOGG-KAMERER $^{1}$, \\ WILKO WEICHERT ${ }^{3}$, BENJAMIN GÖPPERT ${ }^{4}$ and JOHANNES HAYBAECK ${ }^{1,5}$ \\ ${ }^{1}$ Diagnostic and Research Institute of Pathology, Medical University of Graz, Graz, Austria; \\ ${ }^{2}$ Institute of Dermatology and Venerology, Medical University of Graz, Graz, Austria; \\ ${ }^{3}$ Institute of Pathology, Technical University Munich, Munich, Germany; \\ ${ }^{4}$ Department of General Pathology and Anatomy of the Pathology Institute, \\ University Hospital Heidelberg, Heidelberg, Germany; \\ ${ }^{5}$ Department of Pathology, Neuropathology and Molecular Pathology, \\ Medical University of Innsbruck, Innsbruck, Austria
}

\begin{abstract}
Background/Aim: Pancreatic cancer is one of the deadliest forms of cancer and ranks among the leading causes of cancer-related death worldwide. The most common histological type is ductal adenocarcinoma (PDAC), accounting for approximately $95 \%$ of cases. Deregulation of protein synthesis has been found to be closely related to cancer. The rate-limiting step of translation is initiation, which is regulated by a broad range of eukaryotic translation initiation factors (eIFs). Patients and Methods: Human PDAC samples were biochemically analyzed for the expression of various eIF subunits on the protein level (immunohistochemistry, immunoblot analyses) in 174 cases of PDAC in comparison with non-neoplastic pancreatic tissue $(n=10)$. Results: Our investigation revealed a significant down-regulation of four specific eIF subunits, namely eIF1, eIF2D, eIF3C and eIF6. Concomitantly, the protein (immunoblot) levels of eIF1, eIF2D, eIF3C and eIF6 were reduced in PDAC samples as compared with non-neoplastic pancreatic tissue. Conclusion: Members of the eIF family are of relevance in pancreatic tumor biology and may play a major role in translational control in PDAC.
\end{abstract}

This article is freely accessible online.

Correspondence to: Prof. Johannes Haybaeck, MD, Ph.D., Department of Pathology, Neuropathology and Molecular Pathology, Medical University of Innsbruck, Müllerstraße 44, A6020 Innsbruck, Austria. Tel: +43 0512900371300, Fax: +49 3916715818, e-mail: johannes.haybaeck@i-med.ac.at

Key Words: Pancreatic cancer, eukaryotic translation initiation factors, $\mathrm{PI} 3 \mathrm{~K} / \mathrm{AKT} / \mathrm{mTOR}$ pathway, biomarker.
Consequently, they might be useful as potential new biomarkers and therapeutic targets in PDAC.

Pancreatic cancer, including ductal adenocarcinoma (PDAC), its most common manifestation, is still one of the deadliest forms of cancer. It usually affects the elderly, with a 5-year survival rate of 5\% that has not improved over the past 20 years (1). PDAC, originates from the exocrine portion of the gland and accounts for approximately $80 \%$ of cases (1). Compared to other hepatobiliary cancers, only little is known about the risk factors and molecular mechanisms underlying PDAC. Several studies have shown that alcohol and nicotine abuse are the main risk factors, although genetic studies have identified a large number of mutations that affect signaling pathways (2). These genetic variations include $K-R A S$, the tumor suppressors $p 53$ and $p 16$, as well as changes in TGF- $\beta$ and WNT/NOTCH signaling (1-4).

However, the cellular alterations promoting carcinogenesis still need to be investigated for their possible function as new diagnostic markers, prognostic tools and novel therapeutic targets.

Translation, the process of protein synthesis crucially needed for cellular homeostasis, is subdivided into four major steps: initiation, elongation, termination and ribosome recycling. Initiation constitutes the rate-limiting step and is concerted by an abundance of eukaryotic translation initiation factors (eIFs), acting in different ways to generate the fully functional ribosomal apparatus with its associated interaction partners, such as the formyl-methionine-tRNA and the consecutively translated messenger RNA (mRNA) (5). Briefly, translation initiation starts with the formation of the 43S-preinitiation complex, consisting of the $40 \mathrm{~S}$ ribosomal subunit, the ternary 
complex (which is composed of the initiating methionyl-tRNA bound to eIF2-GTP), eIF1, eIF1A and eIF3. This assembly consecutively scans the mRNA for the first start codon (AUG) in the $5^{\prime}$ untranslated region (UTR), leading to codonanticodon interaction assisted by eIF1 and eIF1A. The scanning process is further assisted by the heterotrimeric eIF4F complex (eIF4A, eIF4E and eIF4G). Binding to the matching start codon leads to eIF2-GTP hydrolysis and dissociation of the respective eIF2-GDP. Ribosomal subunit joining is mediated through eIF5B, forming the fully functional ribosome (5).

Dysfunction in translational control is an important downstream endpoint of oncogenic pathways, such as the $\mathrm{PI} 3 \mathrm{~K} / \mathrm{AKT} / \mathrm{mTOR}$ and RAS/RAF/MAPK pathways, which support cellular transformation and tumor development (6). In normal cells, these pathways operate as sensors of nutrient availability, stress or energy, adapting ribosome production and gene expression to the cellular conditions. eIFs have recently been discovered to be involved in tumor development in a variety of malignancies $(6,7)$, and have therefore been suggested as potential therapeutic targets with some substances currently under preclinical and early clinical investigation (7-10). Dysregulation of translation initiation and its interaction with the PI3K/AKT/mTOR signaling pathway are just starting to be understood in pancreatic cancer. Previously described alterations include up-regulation of eIF3A, eIF4E and eIF5A, as well as down-regulation of eIF3F (11-16). However, the molecular mechanism regulating eIFs in tumor biology is still not fully understood.

To address this issue, we investigated the involvement of the PI3K/AKT/mTOR signaling cascade and various eIF subunits, focusing on PDAC. For this purpose, we used protein analyses, immunohistochemistry and immunoblotting to characterize the expression pattern of the mTOR signaling members and various eIF subunits.

\section{Materials and Methods}

Ethics statement. The collection and use of human derived pancreas specimens were approved by the Ethics Committee of the Medical University of Graz, Graz, Austria, according to the ethical guidelines of the 1975 Declaration of Helsinki (EK 28-294 ex 15/16). All samples and medical data used in this study were irreversibly anonymized, and their clinical and pathological data are listed in Tables I and II.

Tissue microarray (TMA). A total of 174 formalin-fixed, paraffinembedded patient samples were retrospectively collected from the Institute of Pathology, Technical University Munich, Germany. Hematoxylin-eosin stained (H/E) slides were reviewed by experienced, board certified pathologists (J.H. and I.B.), who confirmed the diagnosis and identified the areas of tumor and non-neoplastic tissue for each tissue microarray core. Tissue cylinders of $1.2 \mathrm{~mm}$ in diameter were punched out from the chosen tumor areas and embedded as TMA in a fresh paraffin block according to a specific pattern. Tissue sections were cut $(4 \mu \mathrm{m})$ and placed on adhesive-coated glass slides followed by immunohistochemical analysis.
Table I. Clinical and pathological characteristics of 174 patients with pancreatic cancer. For each biochemically assessed PDAC patient, the clinical and pathological characteristics, including sex, age, TNM, stage and the presence of positive lymph nodes, are listed.

\begin{tabular}{lccc}
\hline & Total $(\%)$ & Male $(\%)$ & Female $(\%)$ \\
\hline $\mathrm{n}$ & $174(100)$ & $98(56.3)$ & $76(43.7)$ \\
Mean age & 64.14 & 62.44 & 66.33 \\
$\mathrm{pT}$ & & & \\
$1 / 2$ & $35(20.1)$ & $15(15.3)$ & $20(26.3)$ \\
$3 / 4$ & $139(79.9)$ & $83(84.7)$ & $56(73.7)$ \\
$\mathrm{pN}$ & & & \\
0 & $42(24.1)$ & $22(22.4)$ & $20(26.3)$ \\
1 & $132(75.9)$ & $76(77.6)$ & $56(73.7)$ \\
$\mathrm{pM}$ & & & \\
0 & $168(96.6)$ & $93(94.9)$ & $75(98.7)$ \\
1 & $6(3.4)$ & $5(5.1)$ & $1(1.3)$ \\
Grading & $7(4)$ & $4(4.1)$ & $3(3.9)$ \\
1 & $92(52.9)$ & $51(52)$ & $41(54)$ \\
2 & $75(43.1)$ & $43(43.9)$ & $32(42.1)$ \\
3 & & &
\end{tabular}

Immunohistochemistry. IHC was performed on a Ventana Immunostainer XT (Ventana Medical Systems, Tucson, AZ, USA) by heat-induced epitope retrieval (HIER) in cell conditioning solution for $30 \mathrm{~min}$ and an ultra-VIEW universal DAB Detection Kit (Ventana Medical Systems, Tucson, AZ, USA). The primary antibodies were incubated for 30 minutes using different dilutions (Table II). Each core was semi-quantitatively scored, and an intensity score was defined as follows: $0=$ no staining, $1=$ weak staining, $2=$ moderate staining and $3=$ strong staining. Additionally, the percentage of stained tumor cells was determined (0-100\%) and assigned to 5 groups, $0=0 \%, 1=1-49 \%, 3=50-79 \%$ and $4=80-100 \%$. The intensity score $(0-3)$ was then multiplied by the percentage score (0-4), and the values obtained were subdivided as follows: $0=$ no staining, 1-4=weak staining, 5-8=moderate staining, 9$12=$ strong staining, and displayed in stacked plots.

Human pancreatic cancer patient samples. Human pancreas cancer samples $(n=56)$ and non-neoplastic tissue (NNT) $(n=56)$ were provided by the Department of Pathology, Medical University of Magdeburg, Germany. Informed consent was obtained from all patients. All samples were acquired during surgery, immediately snap frozen in liquid nitrogen, and stored at $-80^{\circ} \mathrm{C}$ until protein or RNA extraction.

Protein extraction and western blot analysis. To prepare protein lysates, frozen tissue samples were homogenized with a MagNA Lyser homogenizer (Roche Diagnostics, Risch-Rotkreuz, Switzerland) and lysed in Nonidet-P40-based lysis buffer (0.05 M Tris-HCl, 0.15 $\mathrm{M} \mathrm{NaCl}, 0.5 \% \mathrm{NP}-40,0.1 \mathrm{mM}$ pefabloc, $1 \mathrm{mM}$ DTT, complete mini EDTA-free, PhosSTOP (Roche Austria GmbH, Vienna, Austria). Protein concentrations were determined using Bradford protein assay (Biorad Protein Assay Dye Reagent; BioRad Laboratories GmbH, Munich, Germany). Thirty ng of total protein lysate was loaded onto sodium dodecyl sulphate (SDS)-polyacrylamide gels (30\% acrylamide/bisacrylamide solution; ROTH, Karlsruhe, Germany), subjected to electrophoresis in mini-vertical electrophoresis units 
Table II. Antibodies for immunohistochemistry.

\begin{tabular}{lccc}
\hline Primary antibody & Company & Dilution & Second antibody \\
\hline eIF1 & Sigma Aldrich (HPA043003) (Bavaria, Germany) & $1: 50$ & Rabbit \\
eIF2 $\alpha$ (D7D3) XP & Cell Signaling (\#5324) (Hessen, Germany) & $1: 2000$ & Rabbit \\
eIF3A & Cell Signaling (\#2538) (Hessen, Germany) & $1: 50$ & Rabbit \\
eIF6 & Gene Tex (GTX63642) (Irvine, CA, USA) & $1: 75$ & Rabbit \\
\hline
\end{tabular}

Table III. Primary antibodies for immunoblotting.

\begin{tabular}{lccc}
\hline Primary antibody & Company & Dilution & Second antibody \\
\hline GAPDH & Sigma (A2103) (Bavaria, Germany) & $1: 1000$ & Rabbit \\
eIF1 & Sigma (HPA043003) (Bavaria, Germany) & $1: 500$ & Rabbit \\
eIF2 $\alpha$ (D7D3) XP & Cell Signaling (\#5324) (Hessen, Germany) & $1: 1000$ & Rabbit \\
eIF3C & Cell Signaling (\#2068) (Hessen, Germany) & $1: 1000$ & Rabbit \\
eIF6 & GeneTex (GTX63642) (Irvine, CA, USA) & $1: 1000$ & Rabbit \\
\hline
\end{tabular}

(Hoefer Inc, Richmond, VA, USA), and blotted onto PVDF membranes (Immobilin-P Transfer Membrane; Millipore, MA, USA) using a Semi Dry Blotting Unit (SCIE-PLAS; Cambridge, England). The membranes were blocked in 5\% non-fat dried milk (AppliChem, Darmstadt, Germany) in $0.1 \%$ TBS tween (TBST) for $1 \mathrm{~h}$ at room temperature. The primary antibodies (Table III) were diluted in $0.1 \%$ TBS tween (TBST) with 5\% BSA and incubated at $4{ }^{\circ} \mathrm{C}$ overnight. The membranes were washed with $0.1 \%$ TBST, followed by incubation with a horseradish peroxidase conjugated secondary antibody (anti-mouse 1:3000 and anti-rabbit 1:5000; GE Healthcare Life Sciences, Buckinghamshire, UK). Proteins were visualized using the chemiluminescence Amersham ECL Western Blotting Detection Reagent (GE Healthcare Life Sciences, Buckinghamshire, UK) and the Image Quant LAS 500 (GE Healthcare, Little Chalfont, UK) detection system. Signals were normalized to the Glyceraldehyde-3phosphate dehydrogenase (GAPDH) loading control (mAb dilution 1:1000, Cell Signaling, Frankfurt, Germany). Densitometric analysis was performed using ImageJ software.

Statistical analysis. The Cancer Genome Atlas (TCGA) public dataset, including 125 PDCA subjects, was analyzed to identify the association between gene expressions stratified by the median survival. Kaplan-Meier curves were generated using the survival $\mathrm{R}$ package. The log rank test was applied to test for an association of survival and gene expression. All results were expressed as mean \pm standard deviation (SD). Differences between groups were assessed using Students $t$-test or Mann-Whitney $U$-test based on data distribution. $p<0.05$ was considered as statistically significant. Statistical analysis and graph generation were performed using GraphPad PRISM version 5.0 (GraphPad software Inc., La Jolla, CA, USA).

\section{Results}

eIF 1, eIF2D, eIF3C and eIF6 are down-regulated in PDAC patients. To adress the relevance of eIF1, eIF2D, eIF3C and eIF6 in PDAC, we investigated their expression by immunohistochemistry (IHC) on tissue microarrays (TMA) in comparison with non-neoplastic tissue (NNT) (Figure 1). In PDCA, 174 patient samples were analyzed, and 10 NNT pancreatic tissues served as controls. Clinical data of patients analyzed by IHC are listed in Table I. Staining was mainly observed in the cytosol. Down-regulation of eIF1 was demonstrated in well and poorly differentiated samples $(p<0.0001)$ (Figure 1A, B and I). eIF2D was found to be down-regulated at the same significance level $(p<0.0001)$, while significant differences were present between well $(\mathrm{G} 1 / 2)$ and poorly (G3) differentiated samples $(p=0.0119)$ (Figure 1C, D, J and 2B). Furthermore, expression of eIF3C was mitigated in tumor samples, independent of differentiation $(p<0.0001)$ (Figure 1E, F, K and 2C). Finally, eIF6 was also down-regulated in PDAC (Figure 2D) as compared with NNT, $(p=0.0083$ ) (Figure 1G, H, L, and 2D). Analysis of staining intensity in PDAC G1/G2 and G3 tumors revealed significant differences in eIF1, eIF2D, eIF3C and eIF6 expression within these groups. eIF1 expression displayed a significant decrease in $\mathrm{G} 1 / \mathrm{G} 2$ $(p<0.0001)$ and high grade G3 tumors $(p<0.0001)$ as compared with NNT (Figure 2A). eIF2D staining was also significantly decreased in $\mathrm{G} 1 / \mathrm{G} 2(p<0.0001)$ and high grade G3 tumors $(p<0.0001)$ as compared with NNT (Figure 2B). When comparing G1/2 ( $p<0.05)$ versus $\mathrm{G} 3$ $(p<0.05)$, significant changes were observed (Figure $2 \mathrm{~B})$ among the different grades. For eIF3C, we noted a significant decrease in the expression for $\mathrm{G} 1 / \mathrm{G} 2$ $(p<0.0001)$ and high grade G3 tumors $(p<0.0001)$ as compared with NNT (Figure 2C). Analysis of staining intensity in PDAC G1/2 and G3 tumors revealed significant differences in eIF6 expression within these groups. eIF6 expression displayed a significant decrease in $\mathrm{G} 1 / \mathrm{G} 2$ 


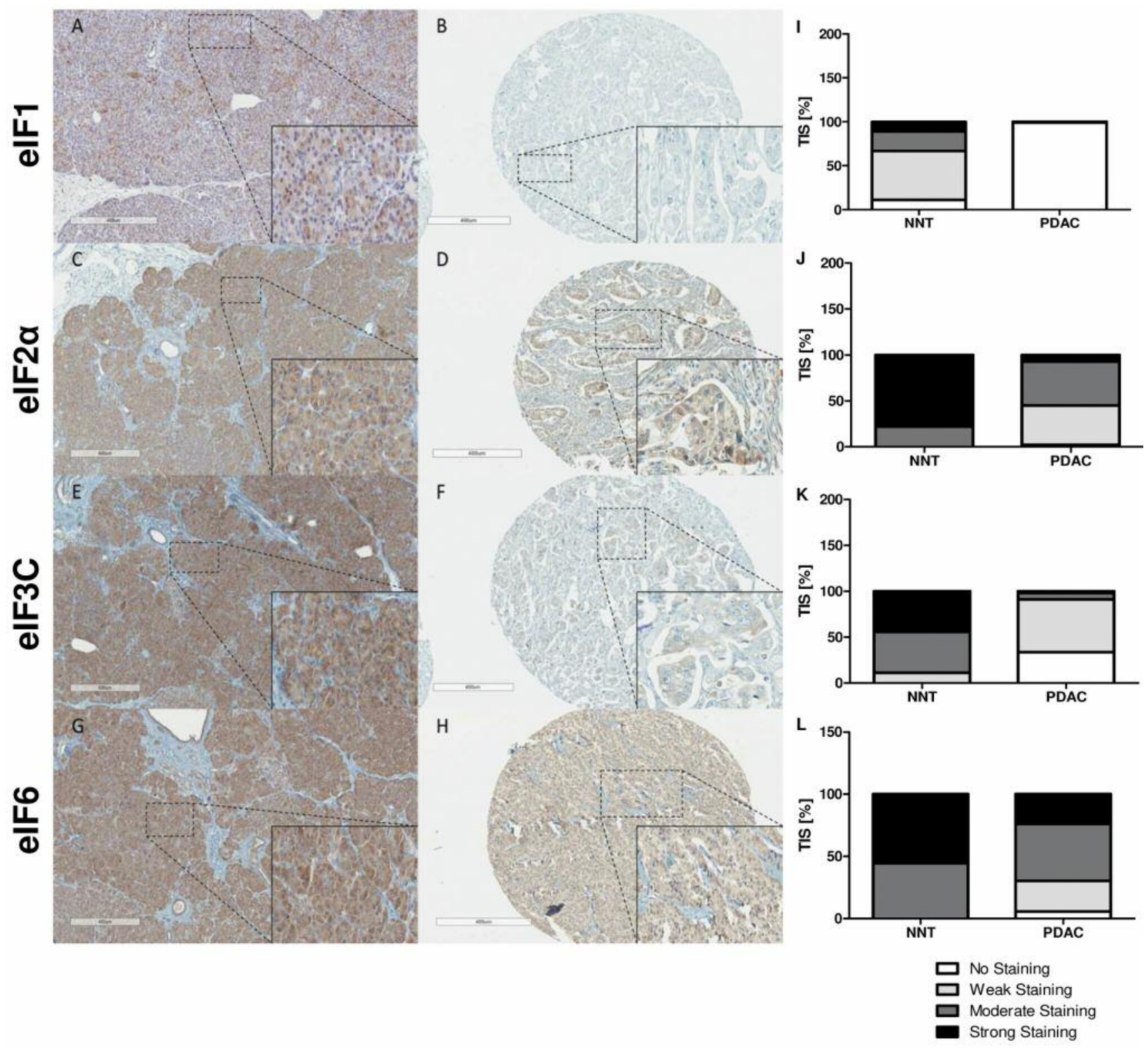

Figure 1. eIF1, eIF2D, eIF3C and eIF6 expression in PDAC in comparison with NNT. (A) Representative pictures of immunohistochemical staining of eIF1 in non-neoplastic tissues (NNT). (B) Representative pictures of immunohistochemical staining of eIF1 in PDAC. (I) Densitometric analyses of immunohistochemical staining of eIF1 in PDAC in comparison with NNT. (C) Representative pictures of immunohistochemical staining of eIF2D of NNT. (D) Representative pictures of immunohistochemical staining of eIF2D in PDAC. (J) Densitometric analyses of immunohistochemical staining of eIF2D in PDAC in comparison with NNT. (E) Representative pictures of immunohistochemical staining of eIF3C in NNT. (F) Representative pictures of immunohistochemical staining of eIF3C in PDAC. (K) Densitometric analyses of immunohistochemical staining of eIF3C in PDAC in comparison with NNT. $(G)$ Representative pictures of immunohistochemical staining of eIF6 in NNT. (H) Representative pictures of immunohistochemical staining of eIF6 in PDAC. (L) Densitometric analyses of immunohistochemical staining of eIF6 PDAC compared to NNT. Scale bars: $400 \mu \mathrm{m}$.

tumors $(p<0.05)$ and high grade $\mathrm{G} 3$ tumors $(p<0.01)$ as compared with NNT (Figure 2D).

Low eIF2D, eIF3C and eIF6 expression correlates with better overall survival of PDAC patients. Based on the results shown above, we investigated the correlation between the expression of eIF1, eIF2D, eIF3C and eIF6 and patients' overall survival. An in silico analysis, including 125 patients of The Cancer Genome Atlas (TCGA) database, was performed. To determine the statistical 

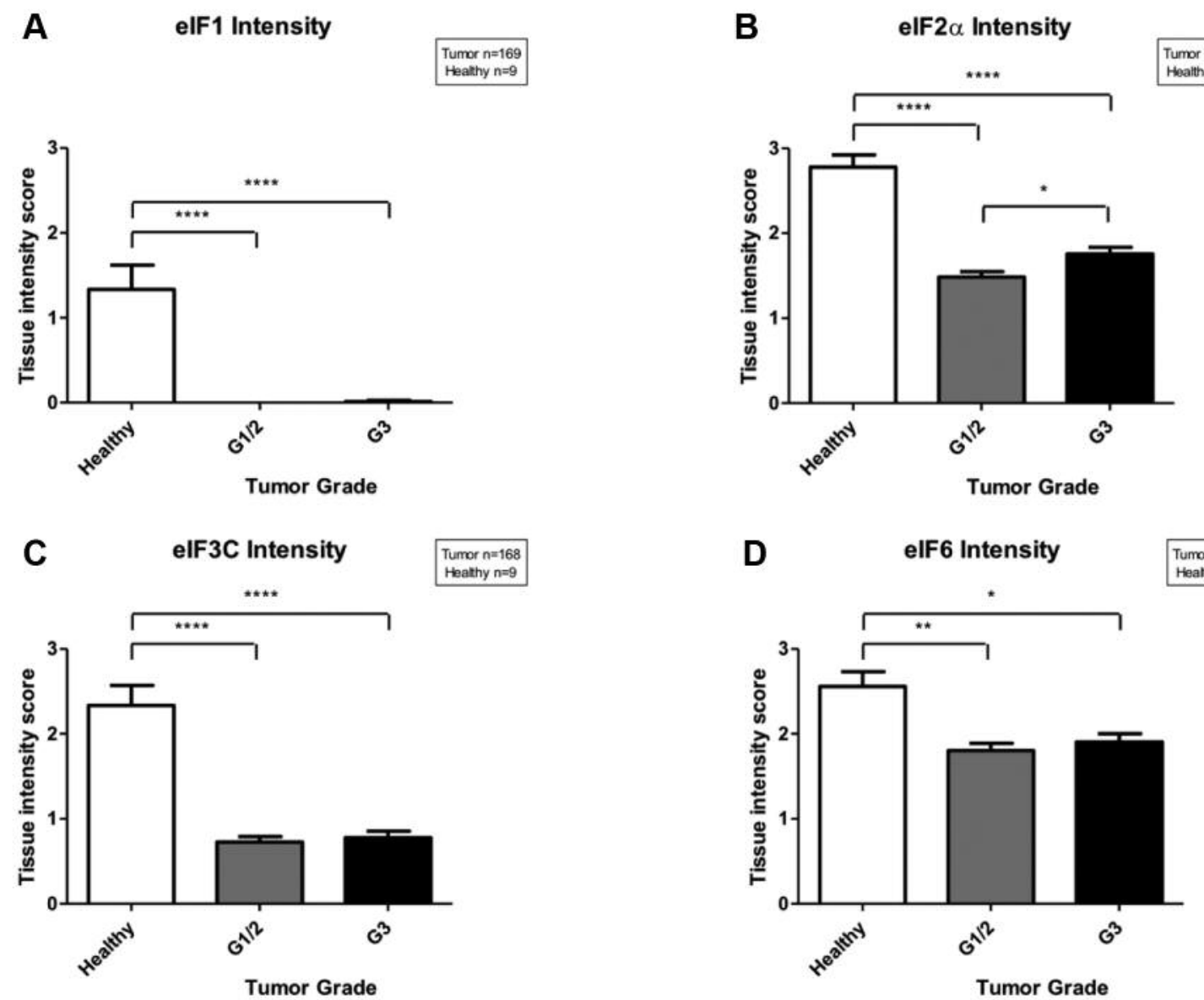

Figure 2. eIF1, eIF2D, eIF3C and eIF6 expression in sub-grouped PDAC samples (well and poorly differentiated) compared to NNT. (A) Expression analyses of immunohistochemical staining of eIF1 in sub-grouped well and poorly differentiated PDAC samples compared to non-neoplastic tissues $(N N T)$. (B) Expression analyses of immunohistochemical staining of eIF2D in sub-grouped well and poorly differentiated PDAC samples compared to non-neoplastic tissues (NNT. (C) Expression analyses of immunohistochemical staining of eIF3C in sub-grouped well and poorly differentiated PDAC samples compared to NNT. (D) Expression analyses of immunohistochemical staining of eIF6 in sub-grouped well and poorly differentiated PDAC samples compared to NNT. Bars represent mean \pm SEM. ${ }^{*} p<0.05$, ** $p<0.01$, ***p $<0.001$, $* * * p<0.0001$. Statistical analysis: Mann-Whitney U-test.

significance, the log rank test was carried out. KaplanMeier curves were generated to assess a potential association of eIF1, eIF2D, eIF3C and eIF6 expression with overall survival in PDAC patients. Lower expression of $e I F 2 D(p=0.002), e I F 3 C(p=0.026)$ and eIF6 $(p=0.132)$ in PDAC was associated with better overall survival as compared with high expression of $e I F 2 D$, eIF $3 C$ and $e I F 6$ (Figure 3B, C and D).

Additionally, the influence of eIF1 on overall survival of PDAC patients was investigated (Figure 3A). Lower expression of $e I F 1$ in PDAC indicated a significantly poorer overall survival as compared with high eIFl $(p=0.048)$ expression. These findings suggest that eIF1, eIF2D, eIF3C and eIF6 might have a different function and might serve as novel prognostic markers for the overall survival of PDAC patients.
eIF 1, eIF 2D, eIF3C and eIF6 are markers of PDAC with good prognosis. To examine whether eIF1, eIF2D, eIF3C and eIF6 protein levels are down-regulated in fresh frozen pancreatic tumor tissue compared to NNT, we prepared protein lysates of 56 PDAC tissue samples and 56 NNT. The lysates were analyzed by immunoblotting. eIF1 demonstrated down-regulation in tumor samples $(p<0.001)$ when compared with NNT (Figure 4A and B). A significant downregulation of eIF2 $\alpha$ was also detected $(p<0001)$ when compared with NNT (Figure 4A and C). Furthermore, expression of eIF3C was lower in tumor samples $(p<0.001)$ as compared with NNT (Figure 5A and B). Finally, eIF6 showed significant differences $(p<0.001)$ between NNT and tumor samples on the protein level (Figure 5A and $\mathrm{C}$ ). Densitometric evaluation (eIF1; eIF2D; eIF3C and eIF6/ GAPDH) of all samples analyzed by immunoblotting is 

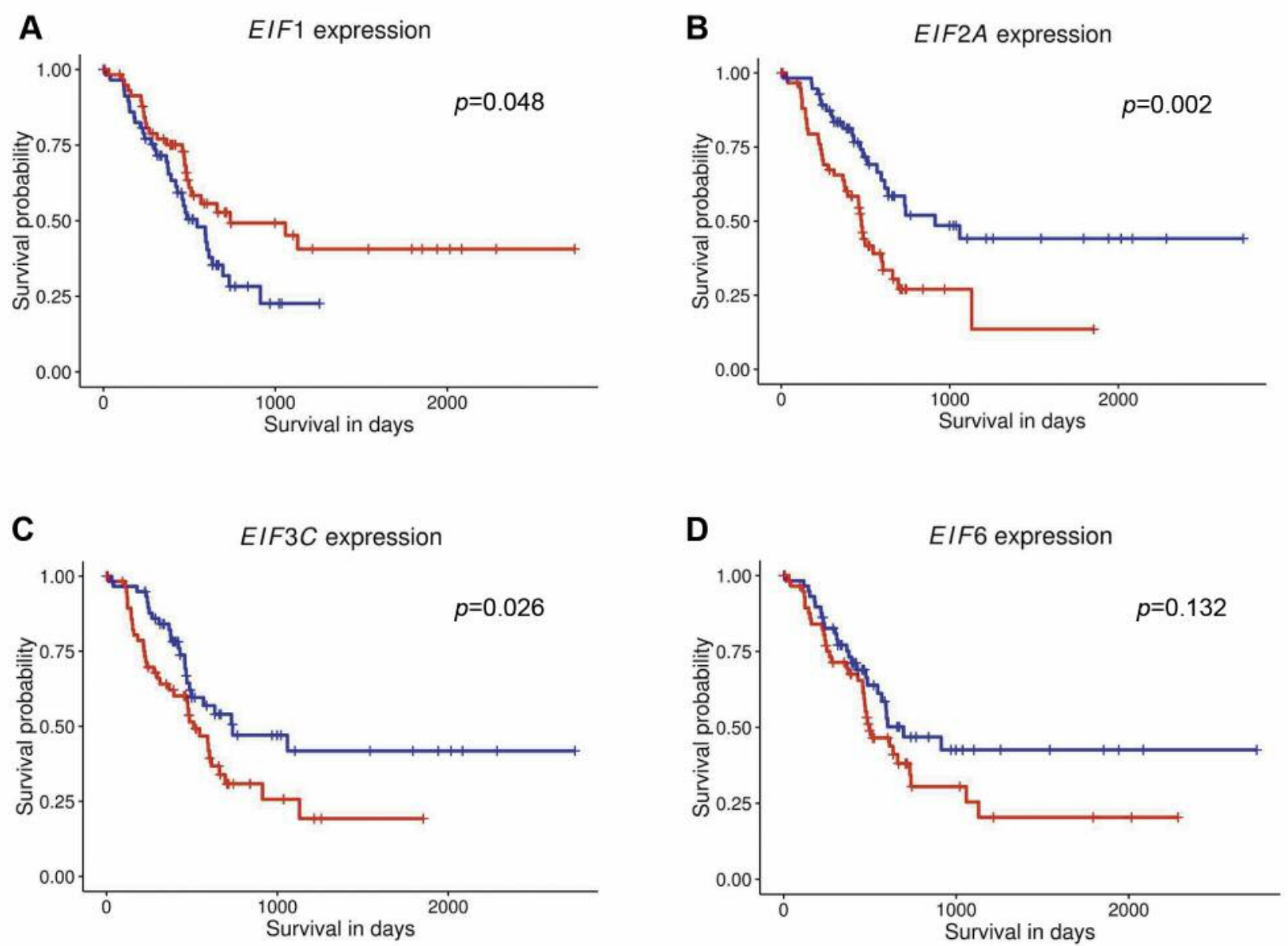

Figure 3. eIF1, eIF2D, eIF3C and eIF6 are clinically relevant candidates in PDAC. (A) Kaplan-Meier curves reflect the effect of eIF1 expression on overall survival for PDAC. Cases are divided into low or high expression according to whether expression is below or above median, and survival was compared using the log-rank test. (B) Kaplan-Meier curves reflecting the effect of eIF2D expression on overall survival for PDAC. Cases are divided into low or high expression according to whether expression was below or above median, and survival was compared using the log-rank test. (C) Kaplan-Meier curves reflecting the effect of eIF3C expression on overall survival for PDAC. Cases are divided into low or high expression according to whether expression was below or above median, and survival was compared using the log-rank test. (D) Kaplan-Meier curves reflecting the effect of eIF6 expression on overall survival for PDAC. Cases were divided into low or high expression according to whether expression was below or above median, and survival was compared using the log-rank test.

shown in Figures 4B, C and 5B and C. These data suggest that eIF1, eIF2D, eIF3C and eIF6 may play an important role during tumorigenesis of PDAC.

\section{Discussion}

Accounting for $95 \%$ of pancreatic cancers, PDAC is still one of the deadliest forms of cancer. It usually affects the elderly, with an overall 5-year survival rate of $8 \%$ that has not improved during the past 20 years (1). Biomarkers to monitor disease progression and novel therapeutic alternative targets for these tumors are strongly required.

eIFs are involved in the translation of growth factors, in proteins influencing cell cycle, growth, as well as in apoptosis and malignant transformation. A broad range of cancer types, including pancreatic cancer, have been candidates for targeting the PI3K/AKT/mTOR pathway due to its common deregulation via amplification, mutation or loss of key regulators.

eIFs are overexpressed in various malignancies, such as squamous cell carcinoma of the head and neck, lung, thyroid, breast and colorectal cancer (17-19). However, data on eIFs in PDAC is still limited.

We presented evidence that compared with normal pancreatic tissue, eIF1, eIF2D, eIF3C and eIF6 are downregulated in PDAC, and are predictors for overall survival in PDAC patients.

The in silico analysis of The Cancer Genome Atlas (TCGA) database revealed a significant influence of eIF2D, 

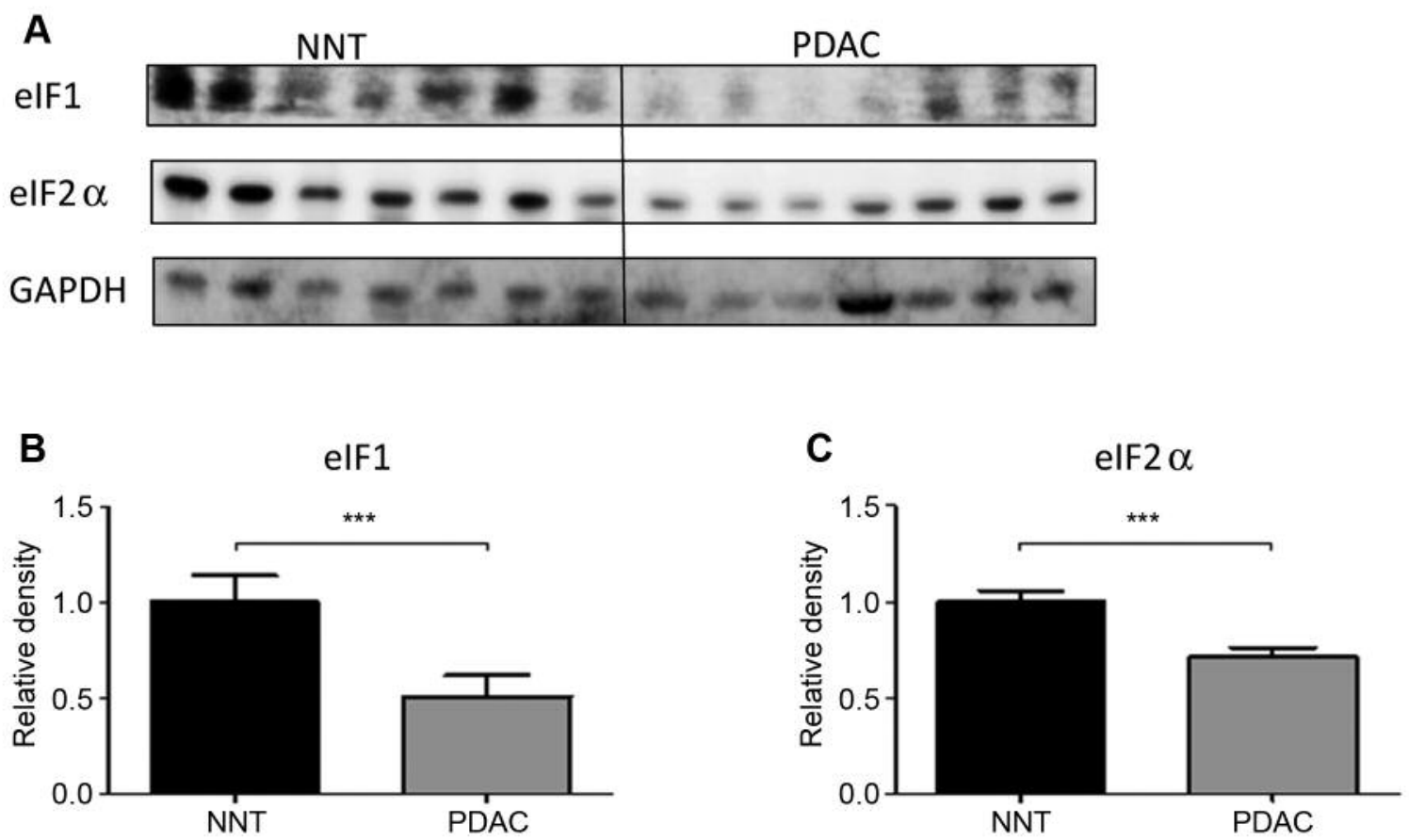

Figure 4. eIF expression in PDAC samples ( $n=56)$ versus non-neoplastic pancreatic tissue $(n=56)$ on protein level. (A) Graph shows immunoblot analysis for PDAC patients as compared with NNT. Equal amounts of protein were resolved on SDS PAGE and immunoblotted with eIF1, eIF2D and GAPDH (loading control) antibodies. (B) Densitometric analyses of immunoblot of eIF1 in PDAC compared with NNT. (C) Densitometric analyses of immunoblot of eIF $2 D$ in PDAC compared with NNT. Bars represent mean \pm SEM. ${ }^{*} p<0.05, * * p<0.01$, ***p<0.001. Statistical analysis: Two-way ANOVA with Bonferroni post-test.

eIF3C and eIF6 on overall survival of PDAC patients. The TCGA analysis revealed a significantly longer overall survival in patients with low eIF2D, eIF3C and eIF6 expression. Moreover, IHC staining of PDAC compared to NNT for eIF1, eIF2D, eIF3C and eIF6, revealed their downregulation in the cytoplasm of pancreatic tumor cells, whereas staining intensity and density were higher in NNT. This decrease in cytoplasmic eIF1, eIF2D, eIF3C and eIF6 in human FFPE tissue specimens has not yet been reported. However, there was no change in the expression patterns of eIF1, eIF2D, eIF3C and eIF6 in the nucleus when comparing PDAC tissues and the non-neoplastic tissues. Our study is the first to provide evidence that eIF1, eIF2D, eIF3C and eIF6 are down-regulated in PDAC compared to nonneoplastic pancreatic tissue, and that they are predictors for overall survival in PDAC.

eIF2D is an important target of regulation, and its activity is often rate-limiting for protein synthesis $(20,21)$. Initiation of mRNA translation requires eIF2-bound GTP and the initiator methionyl-tRNA, leading to the formation of a ternary complex associated with the 40S ribosomal subunit and the initiation factors eIF1, eIF1A and eIF3, resulting in the formation of the $43 \mathrm{~S}$ pre-initiation complex (20). In stressed cells, increased eIF2D switches eIF2 from a substrate to a completive inhibitor of eIF2B, resulting in a global inhibition of protein synthesis (22). Previously, transient eIF2D expression was increased in healthy cells, whereas constitutive overexpression supported tumor initiation and progression (7). Our study also displayed increased expression of eIF1, eIF2D, eIF3C and eIF6 in healthy cells compared to PDAC. eIF6 affects the maturation of $60 \mathrm{~S}$ ribosomal subunits (23) as well as formation of the $80 \mathrm{~S}$ ribosome as anti-association factor (24). It can be found in the nucleolus and in the cytoplasm of a cell. eIF6 was overexpressed in various cancer types, for example metastatic colorectal cancer (17) or non-small cell lung cancer (19). eIF6 expression was indicated to limit cell growth and transformation (25). Regulation of eIF6 is controlled neither transcriptionally via c-Myc nor posttranscriptionally via PI3K/AKT/mTOR signaling (24). The specific molecular mechanisms underlying the role of eIF6 in these processes remains unclear (26-31). Thus, reduced expression of eIF6 and or inhibition of its activity may be used as a biomarker in PDAC. Furthermore, we identified eIF1, eIF21, eIF3C and eIF6 as promising novel targets for PDAC therapies as they were down-regulated on the proteinlevel and, except for eIF2, eIF3C and eIF6, also affected patients' overall survival. 

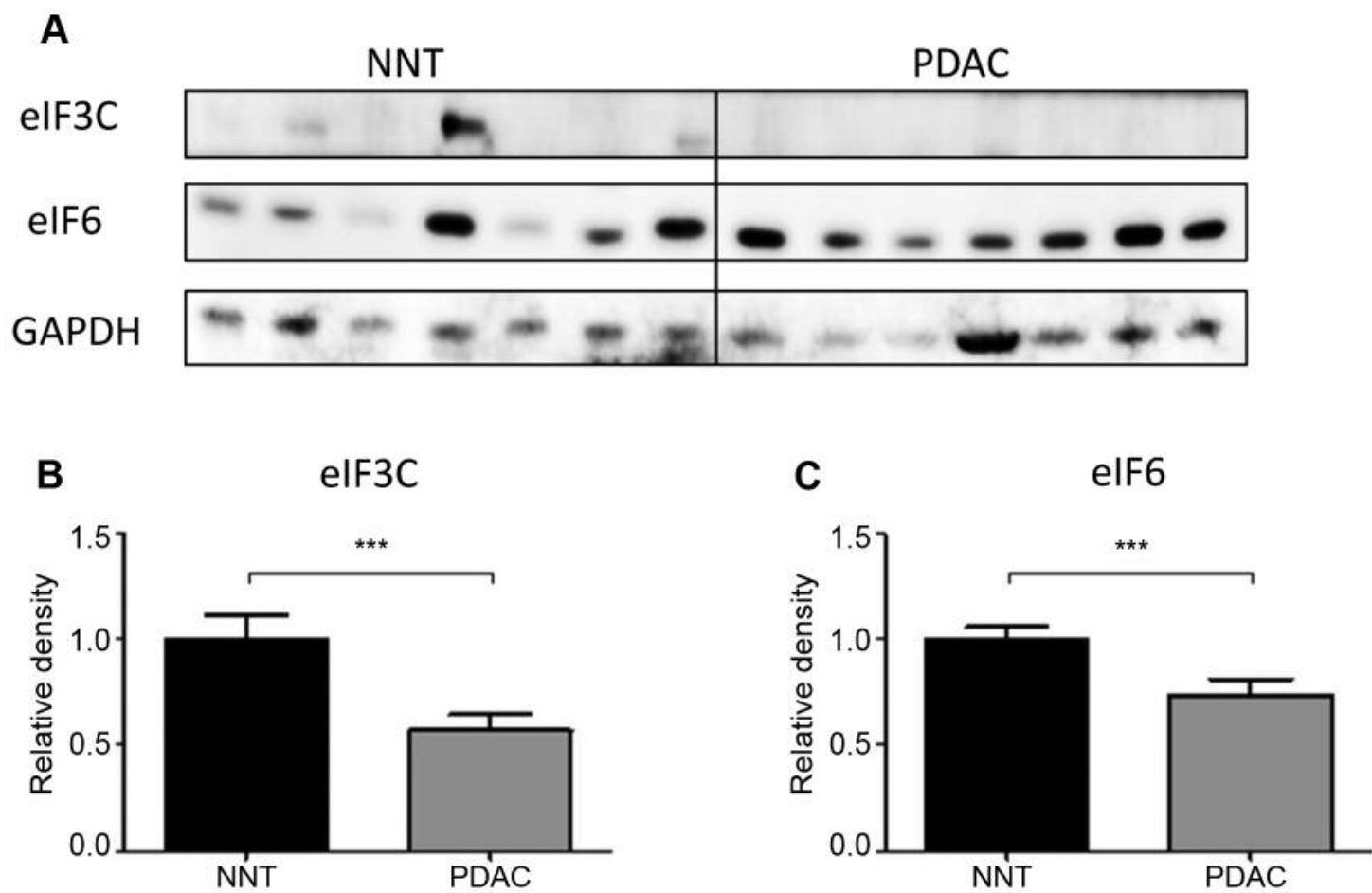

Figure 5. eIF expression in PDAC samples $(n=56)$ versus non-neoplastic pancreatic tissue $(n=56)$ on protein level. (A) Graph shows immunoblot analysis for PDAC patients compared to NNT. Equal amounts of protein were resolved on SDS PAGE and immunoblotted with eIF3C, eIF6 and GAPDH (loading control) antibodies. (B) Densitometric analyses of immunoblot of eIF3C in PDAC compared with NNT. (C) Densitometric analyses of immunoblot of eIF6 in PDAC compared with NNT. Bars represent mean $\pm S E M . * p<0.05, * * p<0.01, * * * p<0.001$. Statistical analysis: Two-way ANOVA with Bonferroni post-test.

Although our study provides promising results, further analyses are required to elucidate the role of eIFs in pancreatic carcinogenesis in more detail. Future experiments should focus on tumor cell type-specific expressions of eIFs under consideration of tumor heterogeneity.

\section{Conclusion}

In conclusion, we suggest that eIF1, eIF2D, eIF3C and eIF6 might serve as prognostic biomarkers for overall survival in PDAC patients, and that their regulation could serve as a potential new therapeutic approach in PDAC. Still, there is a need for future investigations of eIF1, eIF2D, eIF3C and eIF6 expression in PDAC to determine whether eIF1, eIF2D, eIF3C and eIF6 drive pancreatic carcinogenesis.

\section{Conflicts of Interest}

All Authors have no conflicts of interest to declare regarding this study.

\section{Authors' Contributions}

NGS, PP and JH were responsible for the concept and design of this study. NGS, PP, and MGK performed the experiments and procedures. NGS and PP wrote the article. WW and BG participated in building up the study patient cohort. NGS, PP, MGK, WW, BG and $\mathrm{JH}$ commented on the manuscript and interpreted data during the course of the project. JH acquired funding for conducting the entire study. All Authors read and approved the final manuscript.

\section{Acknowledgements}

This research was supported by the Innovative Medicines Initiative Joint Undertaking under grant agreement $n^{\circ}$ (115234), the resources of which are composed of financial contribution from the European Union's Seventh Framework Program (FP7/2007-2013) and T K1 COMET Competence Center CBmed, which is funded by the Federal Ministry of Transport, Innovation and Technology (BMVIT); the Federal Ministry of Science, Research and Economy (BMWFW); Land Styria (Department 12, Business and Innovation); the Styrian Business Promotion Agency (SFG); and the Vienna Business Agency. The COMET program is executed by the FFG (to J.H.). The COMET program is executed by the FFG (to J.H.). The Authors would like to thank Anna Maria Birkl-Toegelhofer for her help with statistical analysis.

\section{References}

1 Ducreux M, Cuhna AS, Caramella C, Hollebecque A, Burtin P, Goéré D, Seufferlein T, Haustermans K, Van Laethem JL, 
Conroy T and Arnold D; ESMO Guidelines Committee: Cancer of the pancreas: ESMO Clinical Practice Guidelines for diagnosis, treatment and follow-up. Ann Oncol 26: v56-v68, 2015. PMID: 26314780. DOI: 10.1093/annonc/mdv295

2 Lowenfels $\mathrm{AB}$ and Maisonneuve P: Risk factors for pancreatic cancer. J Cell Biochem 95: 649-656, 2005. PMID: 15849724 DOI: $10.1002 /$ jcb.20461

3 Yeo TP: Demographics, epidemiology, and inheritance of pancreatic ductal adenocarcinoma. Semin Oncol 42: 8-18, 2015. PMID: 25726048. DOI: 10.1053/j.seminoncol.2014.12.002

4 Waddell N, Pajic M, Patch AM, Chang DK, Kassahn KS, Bailey P, Johns AL, Miller D, Nones K, Quek K, Quinn MC, Robertson AJ, Fadlullah MZ, Bruxner TJ, Christ AN, Harliwong I, Idrisoglu S, Manning S, Nourse C, Nourbakhsh E, Wani S, Wilson PJ, Markham E, Cloonan N, Anderson MJ, Fink JL, Holmes O, Kazakoff SH, Leonard C, Newell F, Poudel B, Song S, Taylor D, Waddell N, Wood S, Xu Q, Wu J, Pinese M, Cowley MJ, Lee HC, Jones MD, Nagrial AM, Humphris J, Chantrill LA, Chin V, Steinmann AM, Mawson A, Humphrey ES, Colvin EK, Chou A, Scarlett CJ, Pinho AV, Giry-Laterriere M, Rooman I, Samra JS, Kench JG, Pettitt JA, Merrett ND, Toon C, Epari K, Nguyen NQ, Barbour A, Zeps N, Jamieson NB, Graham JS, Niclou SP, Bjerkvig R, Grützmann R, Aust D, Hruban RH, Maitra A, Iacobuzio-Donahue CA, Wolfgang CL, Morgan RA, Lawlor RT, Corbo V, Bassi C, Falconi M, Zamboni G, Tortora G, Tempero MA, Gill AJ, Eshleman JR, Pilarsky C, Scarpa A, Musgrove EA, Pearson JV, Biankin AV and Grimmond SM: Whole genomes redefine the mutational landscape of pancreatic cancer. Nature 518: 495-501, 2015. PMID: 25719666. DOI: 10.1038/nature14169

5 Jackson RJ, Hellen CUT and Pestova TV: The mechanism of eukaryotic translation initiation and principles of its regulation. Nat Rev Mol Cell Biol 11: 113-127, 2010. PMID: 20094052. DOI: $10.1038 / \mathrm{nrm} 2838$

6 Pestova TV and Kolupaev VG: The role of individual eukaryotic translation initiation factors in ribosomal scanning and initiation codon selection. Genes Dev 16: 2906-2922, 2002. PMID: 12435632. DOI: $10.1101 / \mathrm{gad} .1020902$

7 Silvera D, Formenti SC and Schneider RJ: Translational control in cancer. Nat Rev Cancer 10: 254-266, 2010. PMID: 29959193. DOI: $10.1101 /$ cshperspect.a032896

8 Spilka R, Ernst C, Mehta AK and Haybaeck J: Eukaryotic translation initiation factors in cancer development and progression. Cancer Lett 340(1): 9-21, 2013. PMID: 23830805. DOI: $10.1016 /$ j.canlet.2013.06.019

9 Blagden SP and Willis AE: The biological and therapeutic relevance of mRNA translation in cancer. Nat Rev Clin Oncol 8: 280-291, 2011. PMID: 21364523. DOI: 10.1038/nrclinonc.2011.16

10 Wang S, Liu Y, Yao M and Jin J: Eukaryotic translation initiation factor 3a (eIF3a) promotes cell proliferation and motility in pancreatic cancer. J Korean Med Sci 31: 1586, 2016. PMID: 27550487. DOI: $10.3346 / \mathrm{jkms} .2016$

11 Fujimura K, Choi S, Wyse M, Strnadel J, Wright T and Klemke R: Eukaryotic translation initiation factor 5A (EIF5A) regulates pancreatic cancer metastasis by modulating RhoA and Rhoassociated kinase (ROCK) protein expression levels. J Biol Chem 290: 29907-29919, 2015. PMID: 26483550. DOI: 10.1074/jbc.M115.687418

12 Kumar, K, Chow CR, Ebine K, Arslan AD, Kwok B, Bentrem DJ, Eckerdt FD, Platanias LC and Munshi HG: Differential regulation of ZEB1 and EMT by MAPK-interacting protein kinases (MNK) and eIF4E in pancreatic cancer. Mol Cancer Res 14: 216-227, 2016. PMID: 26609108. DOI: 10.1158/15417786.MCR-15-0285

13 Fujimura K, Wright T, Strnadel J, Kaushal S, Metildi C, Lowy AM, Bouvet M, Kelber JA and Klemke RL: A hypusine-eIF5APEAK1 switch regulates the pathogenesis of pancreatic cancer. Cancer Res 74: 6671-6681, 2014. PMID: 25261239. DOI: $10.1158 / 0008-5472$

14 Martineau Y, Mueller D, Lasfargues C, Khawand S EL, Anesia $\mathrm{R}$, Pelletier J and Bousquet Ca: Pancreatic tumours escape from translational control through 4E-BP1 loss. Oncogene 33: 13671374, 2014. PMID: 23563181. DOI: 10.1038/onc.2013

15 Doldan A, Chandramouli A, Shanas R, Bhattacharyya A, Leong SPL, Nelson MA and Shi J: Loss of the eukaryotic initiation factor $3 \mathrm{f}$ in pancreatic cancer. Mol Carcinog 47: 235244, 2008. PMID: 18381585. DOI: $10.1002 / \mathrm{mc} .20436$

16 Strnadel J, Choi S , Fujimura K, Wang H, Zhang W, Wyse M, Wright T, Gross E, Peinado C, Park HW, Bui J, Kelber J, Bouvet M, Guan KL and Klemke RL: eIF5A-PEAK1 signaling regulates YAP1/TAZ protein expression and pancreatic cancer cell growth. Cancer Res 77: 1997-2007, 2017. PMID: 28381547. DOI: 10.1158/0008-5472

17 Golob-Schwarzl N, Schweiger C, Koller C, Krassnig S, Goggkamerer M, Gantenbein N, Toeglhofer AM, Wodlej C, Bergler H, Pertschy B, Uranitsch S, Holter M, El-Heliebi A, Fuchs J, Punschart A, Stiegler P, Keil M, Hoffmann J, Henderson D, Lehrach H, Reinhard C, Regenbrecht C, Schicho R, Fickert P, Lax S and Haybaeck J: Separation of low- and high-grade colon and rectum carcinoma by eukaryotic translation initiation factors 1, 5 and 6. Oncotarget 8(60): 101224-101243, 2017. PMID: 29254159. DOI: $10.18632 /$ oncotarget.20642.

18 Wang XL, Cai HP, Ge JH and Su XF: Detection of eukaryotic translation initiation factor $4 \mathrm{E}$ and its clinical significance in hepatocellular carcinoma. World J Gastroenterol 18: 2540-2544, 2012. PMID: 22654452. DOI: 10.3748/wjg.v18.i20.2540

19 Gantenbein N, Bernhart E, Anders I, Golob-Schwarzl N, Krassnig S, Wodlej C, Gogg-Kamerer M, Lindenmann J, Fink-Neuböck N, Brcic L, Gollowitsch F, Stacher-Priehse E, Rolff J, Hoffmann J, Silvestri A, Regenbrecht C, Reinhard C, Pehserl AM, Pichler M, Sokolova O, Naumann M, Mitterer V, Pertschy B, Bergler H, Popper H, Sattler W and Haybaeck J: Eukaryotic translation initiation factor 6 impacts NSCLC Eur J Cancer 101: 165-180, 2018. PMID: 30077122. DOI: 10.1016/j.ejca.2018.07.001

20 Sonenberg $\mathrm{N}$ and Hinnebusch AG: Regulation of translation initiation in eukaryotes: mechanisms and biological targets. Cell 136: 731-745, 2009. PMID: 19239892. DOI: 10.1016/j.cell. 2009.01 .042

21 Wek RC, Jiang HY and Anthony TG: Coping with stress: eIF2 kinases and translation-al control. Biochem Soc Trans 34: 7-11, 2006. PMID: 16246168. PMID: 16246168. DOI: $10.1042 / B S T$ 20060007

22 Proud CG: Regulation of eukaryotic initiation factor eIF2B. Prog Mol Subcell Biol 26: 95-114, 2001. PMID: 11575168. DOI: 10.1007/978-3-642-56688-2_4

23 Sanvito F, Vivoli F, Gambini S, Santambrogio G, Catena M, Viale E, Veglia F, Donadini A, Biffo S and Marchisio PC: Expression of a highly conserved protein, p27 BBP, during the progression of human colorectal cancer. Cancer Res 60: 510516, 2000. PMID: 10676626 
24 Miluzio A, Beugnet A, Volta V and Biffo S: Eukaryotic initiation factor 6 mediates a continuum between $60 \mathrm{~S}$ ribosome biogenesis and translation. EMBO Rep 10: 459-465, 2009. PMID: 19373251. DOI: 10.1038/embor.2009.70.

25 Basu U, Si K, Warner JR and Maitra U: The Saccharomyces cerevisiae TIF6 gene encoding translation initiation factor 6 is required for 60 S ribosomal subunit biogenesis. Society 75: 14531462, 2001. PMID: 11238882. DOI: 10.1128/MCB.21.5.14531462.2001

26 Parsyan A, Svitkin Y, Shahbazian D, Gkogkas C, Lasko P and Merrick WC: mRNA helicases: The tacticians of translational control. Nat Rev Mol Cell Biol 12(4): 235-245, 2011. PMID: 21427765. DOI: $10.1038 / \mathrm{nrm} 3083$

27 Siddiqui N and Sonenberg N: Signalling to eIF4E in cancer. Biochem Soc Trans 43(5): 763-772, 2015. PMID: 26517881. DOI: $10.1042 / B S T 20150126$

28 Miluzio A, Beugnet A, Grosso S, Brina D, Mancino M, Campaner S, Amati B, de Marco A and Biffo S: Impairment of cytoplasmic eIF6 activity restricts lymphomagenesis and tumor progression without affecting normal growth. Cancer Cell 19: 765-775, 2011. PMID: 21665150. DOI: 10.1016/j.ccr.2011. 04.018
29 García-Márquez A, Gijsbers A, de la Mora E and Sánchez-Puig $\mathrm{N}$ : Defective guanine nucleotide exchange in the Elongation Factor-Like 1 (EFL1) GTPase by mutations in the ShwachmanDiamond syndrome protein. J Biol Chem 290: 17669-17678, 2015. PMID: 25991726. DOI: 10.1074/jbc.M114.626275

30 Biffo S, Sanvito F, Costa S, Preve L, Pignatelli R, Spinardi L and Marchisio PC: Expression of a highly conserved protein, p27BBP, during the progression of human colorectal cancer. Cancer Res 60(3): 510-516, 2000. PMID: 10676626.

31 Sanvito F, Piatti S, Villa A, Bossi M, Lucchini G, Marchisio PC and Biffo S: The beta4 integrin interactor p27 (BBP/eIF6) is an essential nuclear matrix protein involved in $60 \mathrm{~S}$ ribosomal subunit assembly. J Cell Biol 144: 823-837, 1999. DOI: $10.1083 /$ jcb.144.5.823
Received April 15, 2020

Revised April 23, 2020

Accepted April 28, 2020 